\title{
TO ENHANCE THE PERFORMANCE AND EFFICIENCY
}

\author{
K K Srivastava* \\ Consultant, Pharma and Biotechnology, Gurgaon 122011 (Haryana), India
}

Every one is interested in enhancing the performance of his work force. It is said that the Indian Government, the largest employer of man power in India, is riddled with inefficient and corrupt man power. It has been estimated that hardly 10 per cent of its employees seem to be motivated and have will power to give their best to the Organization to which they serve.

The enhancement in physical and mental work efficiency of man and domesticated animals has played a key role in the evolution of civilization. From the time when muscle power was the only known motive power to the times when man used the wheel, invented powerful machines, used new energy sources, modified and evolved into sophisticated electronic controls, the effort has been to increase the work performance and efficiency. It simply means that the man has limited capacity to work but it can be enhanced. In addition; the capacity to work deteriorates as the man performs in time, he ages, he is under mental distress and the environment in which the work is being done is hostile to his well being. The latter may be due to high and low environmental temperatures, atmospheric pressure such as in high mountains and deep sea, absence of gravity and oxygen as in space, presence of noise and absence of sound and so on. Increasing work performance played a key role in competitive sports all over the world. The means to achieve higher performance in sports had to be well defined and the use of performance enhancing drugs (Table 1) had to be prohibited by legislation. There is a constant tug of war between the sports administration diagnosing the performance enhancers, doping methods and mechanisms and the sports persons and their coaches, dodging them (1). Yet, in many areas, the enhancement in physical and mental performance has been considered

\section{Address for Correspondence :}

\section{Prof. K.K.Srivastava}

Consultant, Pharma and Biotechnology

12/4B Aravali View Rail Vihar, Sector 56,

Gurgaon 122011 (Haryana), India

E-mail : srivastavakaushal@yahoo.co.in
Table 1 : Doping Agents in Professional Sports

Stimulants: Amphetamines, Cocaine, Ephedrine, Strychnine, Phenylpropalamine, Caffeine etc

Narcotic Analgesics : Codeine, Pethidine, Morphine, Dextropropoxyphen.

Corticosteroids : Blood Doping, Artificial Oxygen Carriers

Growth Hormone

Anabolic Steroids : Stanozolone, Methyl Testosterone, Testosterone, DHEA, Tetrahydrogestrinone

$\beta$ Blockers : Atenolol, Propranol,Sotalol

Diuretics : Furosemide, Spiranolactone, Trimeterene

desirable since time immemorial. Ageing is one such area. The loss of physical strength, memory and learning capability are the manifestation of dwindling metabolic machinery. It is desirable to maintain and if possible to restore it in aged population. Similar is the case with the large work force in industries. The development of 'fatigue' and 'boredom', due to continuous and repetitive work in drab ambience is accompanied with loss of millions of man hours and many occupational diseases. A recent ILO estimate says " 4 per cent of world's GDP is lost due to occupational diseases and illness." This needs prevention and if possible restoration of lost strength to carry out the work for longer shift hours. In addition, the use of prosthesis for improving the work performance of physically compromised individuals is highly desirable (2, Table 2).

The enhancement in work performance includes in it, a technique for the quantitative measurement of work otherwise how one will define the utility of a technique or a process as performance enhancer. This depends entirely on the aim of the task being performed. For example if the task requires acquiring a target and retrieval of information from stored data or memory, you need to measure mental performance or the indices for higher cognitive functions such as concentration, perception and vigilance. On the other hand, if you require a performance enhancement in a short race of 100 meters by cutting out the time required, you simply have to measure the time for completing the race in a shortest possible time. In a 


\section{Table 2 : Ethical Issues}

Regulations to prevent acquisition of powers

People do not naturally posses.

Use of Modafinal to prevent sleep deprivation effects

To make disadvantaged persons able

Carbon fibers prosthetics

Enhancement in one, handicap in others.

Brain Prosthesis.

Forgetting has Advantages

marathon race, on the other hand, you are required to increase the endurance of the athlete so that he can run with an optimum speed for a long duration. In a game of skill requiring neuromuscular coordination, one has to determine eye and hand coordination. While doing a job requiring neuromuscular coordination, minimum number of mistakes is to be made while maintaining the speed as in motor driving and piloting any other vehicle. Thus, there could be several parameters, even more than one in a task, which would require to be measured for evaluation of the work performance enhancer. This complicates the field. Target acquisition, mobility, survivability, sustainability and decision making are some of important criteria for work measurement.

I am making a differentiation between mental and physical work performance for the sake of simplifying the issue. Otherwise the two are highly interconnected. Physically healthy only can perform mental tasks efficiently and a healthy mind resides only in a healthy body. Alert mind and physical vitality invariably go together. Though, there are some obvious exceptions such as Stephen Hawking and many compromised individuals.

There are many discrete factors which determine the capacity and capability of the person to do a job. Motivation or the inclination to perform to the best of ones ability is one of such factors. A soldier and a terrorist give away their life to achieve their goal. What are the factors and the methods which bring about this in a person are still the subjects for study and contemplation. The leadership in any areas would give any thing to find out what motivates their work force. Another is Will Power. Many so called impossible tasks of human valor and endurance have been performed due to sheer grit and strong will power in spite of poor physical and challenging conditions. Mountaineering and climbs to Everest are full of many such episodes. The other is the concentration to achieve the desired results. Sufficient arousal to understand and transform the problems into elements of action is necessary for higher efficiency in solving problems.
For achieving higher physical work capacity, it is essential to enhance metabolic capacity to burn high amount of food to get sufficient energy released. At the same time the tissue homeostasis or dynamic metabolic balance in the body tissues especially in the skeletal muscles, has to be maintained. Availability of sufficient energy reserves in the body is another factor having a bearing on the endurance work being performed. In addition, neuromuscular control on the movement of body parts and limbs is highly significant

There are several techniques available for increasing the capacity for work. (3) The most common and popular is exposing the organism to increasing amount of effort to do better than earlier. This is often termed as training and practice. In fact, this is the age old technique of adapting the organism to gradually increasing amount of stressor. Better performance in sports, studies, shop floors, almost every where, can be achieved through this technique.

Along with the adaptation to one type of environment such as hot environment, comes simultaneously adaptation to several other types of environments. The enhanced cardiovascular strength achieved in hot environment brings about simultaneously enhanced exercise tolerance especially of the endurance type. This phenomenon is known as Cross Adaptation. Cross Adaptation enables a healthy person to perform better than others in every sport.

Among the more recently acknowledged techniques, although known since ages, are the New Age Techniques like Yoga and Meditation, specially the Breathing Techniques or Pranayam, for enhancing the physical and mental performance. Yoga including Pranayam brings about strengthening of cardio respiratory system with mental tranquility. This would result in improved neuromuscular coordination and performance in jobs requiring skill and mental effort.

The use of nutrients and nutritional factors in improving endurance, skilled work performance, sports and games and mental work has been acknowledged since ages. Ayurveda, the science and art of living long life to the full, has proper nutrition and life style at its core. Nutraceuticals, though a recently coined word, had its beginning in Ayurveda some 3500 years ago. Russians, some 50 years ago, gave another word, Adaptogens, to nutrients and biologicals derived from plants and animals which were useful in enhancing wok performance in adverse environments, in sports, among soldiers, miners, students, workers and aged individuals.(4) These affected immune system, improved cardio respiratory 
system, mental vasculature and overall physical stamina and mental alertness. The modern scientific instrumentation and techniques have provided tools for the evaluation of such processes and biologicals which affect work performance. The utility of these chemicals, nutraceuticals, biologicals and processes for the welfare of man kind is enormous. All of this now forms a part of the medicine called the "Adaptive Medicine." (Table 3)

\section{Table 3 : Adaptive medicine}

Nutrients and nutritional factors in improving endurance, skilled work performance, sports and games and mental work

Nutraceuticals, had its beginning in Ayurveda, 3500 years ago.

Russians, 50 years ago, gave another word, Adaptogens, to nutrients and biologicals, useful in enhancing wok performance in adverse environments, in sports, among soldiers, miners, students, workers and aged individuals.

These affected immune system, improved cardio respiratory system, mental vasculature and overall physical stamina and mental alertness.

All of this now forms a part of the medicine called the "Adaptive Medicine."

There are several necessary characteristics for a substance to be effective performance enhancer (Table 4). First of all, the substance should have a physiological and metabolic role to play in the tissue concerned viz skeletal muscles, heart and vasculature and brain (5). For example, carnitin and choline have a role to play in skeletal and brain tissue metabolism respectively. Both can play a role in enhancing exercise tolerance and learning. However, both should have a capacity to get through digestion, get absorbed in the digestive tract and get delivered through blood circulation at the site of action. In addition, the tissue concentration of such substances should be adequate to become effective. Thus one gm of carnitin a day taken orally has been found to be an effective exercise enhancer.

Some of the effective performance enhancers are peripheral

Table 4 : Nutritional Physical Performance Enhancers

Central/Peripheral Stimulants

Caffeine, Choline, Branched chain amino acids

Acting/Supplementary Fuels

Sugars, Phosphate Loading, DHAP, Pyruvate, Medium Chain Fatty Acids, Ketone bodies and Polyketones

Diluting Metabolic Byproducts

Bicarbonate, Dichloracetate, Water

Enhancing Recovery

Water and Sugars and central neural stimulants. Caffeine and some of the Branched chain amino acids affect learning, memory and vigilance functions through their neural stimulation effects. Caffeine can enhance physical work performance through improved neuromuscular coordination in an endurance effort. In moderate dosage, it is established to increase alertness, prevent reduction in mental and physical work performance due to sleep deprivation. Choline as lecithin has been found to effective in many animal and human experiments as effective mental work performance enhancer. Among the branched chain amino acids tryptophan has been found to have a calming effect and a sleep inducer. On the other hand, Tyrosine, Phenyl alanine and Glutamic acid enhance arousal and awareness and make the organism alert.Arginine is another amino acid, constituents of several well known adaptogens such as Ginseng, found to be an effective mental function enhancer. It is a precursor of nitric oxide, an endothelium relaxing factor

Carbohydrates and sugars act as primary source of energy in all mental and physical activity. If taken in adequate amounts before or during the physical activity they provide a ready source of energy and if in excess get stored in skeletal muscles and liver as glycogen. A large store of glycogen is essential for spurt activities such as 100 meters run. Increased energy stores are desirable for endurance activities as well such as marathon run. By providing drinks rich in sugars and carbohydrates to sports persons either before the event or during the event, immediate need of energy is sought to be fulfilled.

Supplementary foods containing phosphates and smaller chain fatty acids are also loaded in the athletes to provide them with adequate amounts of secondary source of energy and supplementary nutrients needed for metabolic utilization of sugars. In addition, the metabolic product of intense physical activity such as lactic acid and carbonic acids are sought to be neutralized through bicarbonate, dichloracetate and water intake. During the rest period for jobs requiring intermittent activity, it is also necessary to enhance recovery and shorten the recovery time. Two important constituents required for this, are water and sugars. This avoids the dehydration and exhaustion often seen in jobs requiring intense effort. Recently Polyketones have been developed as supplementary foods for peak performance of soldiers.

Champions have something more in addition to training and adaptation techniques. It is their genes. Several genes have been identified whose expression or silence to endurance has been established at least in experimental animals. Some of 
these are; ACE, APOE, Erythropoietin, Alpha Actin-3, CKMM, Myostatin and PEPCK-C ( Table 5).

\section{Table 5 : Gene Disposition}

\section{About 50 Genes}

ACE; II variant Endurance, Marathon Runners \& Mountaineers

DD Variant Sprinters

Alpha Actinin-3 ; fast muscle fibers

PPAR- Delta; Slow Fibers for Endurance in Mice

CKMM; Maximum Oxygen Ventilation on Training

Myostatin; Large muscles

In spite of the best of science and technology being made available to sportsmen in individual and team events, there has always been an element elusive between success and failure. This has something to do with faith and happiness. To achieve success one has to have faith on one self, on the job being done and on the GOD that a job well done with his guidance and spirit will eventually lead to success. The GOD may mean different things to different people. All are acceptable.

The happiness is an essential ingredient of the recipe for success. There are many ways to achieve happiness. Enjoying the job and work being done is one of them. All successful people enjoy doing their work. Happiness is within. It does not come from outside. But you can train yourself to remain happy even in the worst of the times. Tough times never last, the tough people do. Food and dietary habits play a significant role in generating happiness. This has been recognized since ages. Aggression and Tranquility are the extremes of the diet spectrum. By modifying the diet rich in serotonin uptake inhibitors and serotonin generating tryptophan, you can achieve tranquility and focused concentration. Milk is one such food. On the other hand diets rich in stimulants, create aggression, hostility, hatred and killer tendencies. There are happiness drugs but nobody advises to take them unless one is sick. There are herbs like Acorus calamus, Nordostachys jatamansi etc which affect mental make up (6, Table 6). Similarly Hormones like DHEA, create an ambience of well being and happiness. But these methods are not lasting ones and would require repeated administration. Achieving lasting happiness is an art and science both and it includes life style changes. I would prefer not to do a job which I don't enjoy and feel happy doing. Every one is not lucky enough to be able to choose his job. But every one has a right to look for and prepare for the job one feels happy doing. Even looking for and preparing for a chance to get a job of ones choice is enough of an incentive and motivation to give happiness. Happiness
Table 6 : Mind Conditioners

Food
Tyrosine, Phenyl alanine,
Tryptophane, Choline
Carnitine, Caffeine
Drugs
Prozac,Zologt,Paxil,Welbutrine
Serotonin uptake inhibitors,
Herbal
Acorus calamus,
Nordostachys jatamansi etc
Hormonal
DHEA (Dehydroepiandrosterone)

of the workers and supervisors in any organization is an integral part of the management processes to enhance performance and efficiency. This is the secret of success individually, in a community such as an organization, society and in a comity of Nations. A happy worker is always an asset and happiness breads itself.

* Editorial is based on Awadhesh Saran Memorial Oration Conferred on author who is former Director, Bio Medical Sciences, DRDO, Govt. of India, Professor Emeritus BR Ambedkar Centre for Biomedical Research, Delhi, Chairman Scientific \& Academic Committee ACBI and Member Editorial Board IJCB.

\section{REFERENCES}

1. Sweeney HL "Gene Doping”. Scientific American 2004; 291: 36-43.

2. Alexander Brian. "Is there a human right to be super human?" 24 Aug 2007 www.msnbc.msn.com/id/13054181/from/ET/ print/l/displaymode/1098.

3. Srivastava KK, Kumar R, Grover SK. "Endurance Enhancement in Adverse Environment" 1999, First International Seminar on Force Multiplier Technologies for Naval and Land Warfare, New Delhi, India, October 13-15, 1999, Defense Research and Development Organization, Ministry of Defense, pages 407-15.

4. Brekhman II. "Man and Biologically Active Substances" 1980, Pergamon Press Ltd ,Oxford (England), pages 6-20.

5. Salter CA, Salter C de Lerma. "Age Busters" 2002, Published by Health Harmony New Delhi, p 27-92.

6. Davidson JRT, Connor KM. "Herbs for the Mind" 2002, Published by Health Harmony New Delhi, p 146-90. 